\title{
Title: Neutron Star Evolution and Emission
}

Author(s):

R. I. Epstein, NIS-2

B. C. Edwards, NIS-2

T. J. Haines, P-23

C. M. Hoffman, P-DO

J. A. Guzik, X-TA

J. G. Hills, T-6

K. Lackner, T-3

M. B. Davies, Institute Astron., Cambridge U.

J. A. Goodman, Dept. Phys., U. Maryland

B. McNamara, Dept. Astron., New Mexico State U.

A. V. Olinto, Dept. Astron. and Astrophys., U. Chicago

G. H. Sigel, Dept. Ceramics, Rutgers U.

G. B. Yodh, Dept. Phys., UC-Invine

Submitted to: DOE Office of Scientific and Technical Information (OSTI)

\section{DISCLAIMER}

This report was prepared as an account of work sponsored by an agency of the United States Government. Neither the United States Government nor any agency thereof, nor any of their employees, makes any warranty, express or implied, or assumes any legal liability or responsibility for the accuracy, completeness, or usefulness of any information, apparatus, product, or process disclosed, or represents that its use would not infringe privately owned rights. Reference herein to any specific commercial product, process, or service by trade name, trademark, manufacturer, or otherwise does not necessarily constitute or imply its endorsement, recommendation, or favoring by the United States Government or any agency thereof. The views and opinions of authors expressed herein do not necessarily state or reflect those of the United States Government or any agency thereof.

\section{DISTRIBUTION OF THIS DOCUMENT IS UNLIMITED}

\section{Los Alamos

Los Alamos Natlonal Laboratory, an affirmative action/equal opportunity employer, is operated by the University of Californla for the U.S. Department of Energy under contract W-7405-ENG-36. By acceptance of this article, the publisher recognizes that the U.S. Government retains a nonexclusive, royaltyfree license to publish or reproduce the published form of this contribution, or to albw others to do so, for U.S. Government purposes. Los Alamos National Laboratory requests that the publisher Identity this article as work performed under the auspices of the U.S. Department of Energy. Los Alamos National Laboratory strongly supports academic freedom and a researcher's right to publish; as an institution, however, the Laboralory does nol endorse the viewpoint of a publication or guarantee its technical correctness. 


\section{DISCLAIMIER}

Portions of this document may be illegible in electronic image products. Images are produced from the best available original document. 


\title{
Neutron Star Evolution and Emission
}

\author{
Richard I. Epstein* and Bradley C. Edwards \\ Nonproliferation and International Security Division, Los Alamos National Laboratory \\ Todd J. Haines and Cyrus M. Hoffman \\ Physics Division, Los Alamos National Laboratory \\ Joyce A. Guzik \\ Applied Theoretical and Computational Physics Division, Los Alamos National Laboratory
}

Jack G. Hills and Klaus Lackner

Theoretical Division, Los Alamos National Laboratory

Melvyn B. Davies

Institute of Astronomy, Cambridge University

Jordan A. Goodman

Department of Physics, University of Maryland

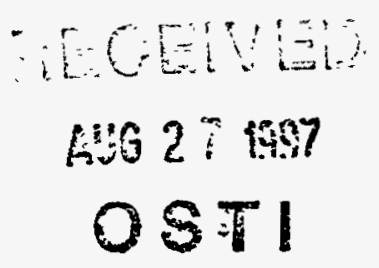

Bernie McNamara

Department of Astronomy, New Mexico State University

Angela V. Olinto

Department of Astronomy and Astrophysics, University of Chicago

George H. Sigel

Department of Ceramics, Rutgers University

Gaurang B. Yodh

Department of Physics, University of California-Irvine

\begin{abstract}
This is the final report of a three-year, Laboratory Directed Research and Development (LDRD) project at the Los Alamos National Laboratory (LANL). We investigated the evolution and radiation characteristics of individual neutron stars and stellar systems. Our work concentrated on phenomena where new techniques and observations are dramatically enlarging our understanding of stellar phenomena. Part of this project was a study of $\mathrm{x}$-ray and gamma-ray emission from neutron stars and other compact objects. This effort included calculating the thermal $x$-ray emission from young neutron stars, deriving the radio and gamma-ray emission from active pulsars and modeling intense gamma-ray bursts in distant galaxies. We also measured periodic optical and infrared fluctuations from rotating neutron stars and search for high-energy $\mathrm{TeV}$ gamma rays from discrete celestial sources.
\end{abstract}

* Principal Investigator, E-mail: repstein@lanl.gov 


\section{Background and Research Objectives}

Two exciting focuses of modern astrophysics are the evolution of stellar systems and the generation of observable radiation by stars. Our work is directed toward addressing critical aspects of these important issues. Part of our research program considers the $\mathrm{x}$-ray and gamma-ray emission from neutron stars and other compact objects. With the development of new $\mathrm{x}$-ray and gamma-ray satellite-based observing systems, the astrophysics community has, for the first time, the ability to observe stellar objects over broad spectral bands and with fine time resolution.

In our theoretical work we model and compute the variable spectra from pulsars' magnetospheres, neutron star surfaces, and gamma-ray bursts. Neutron stars are born as rapidly rotating objects that later slow due to interactions with their environment. We are acquiring optical data on neutron stars from ground based facilities and using these data to measure the periodic fluctuations from stellar rotation and to deduce the initial rotation rates and the subsequent deceleration.

One of the most exciting areas in high-energy astrophysics involves gamma rays from compact stars. These emissions have been reported by more than eight groups throughout the world, but their exact nature, and even their existence, is still an open question. We have launched an extensive search for these gamma rays using the most advanced scintillator array and Cherenkov detectors..

\section{Importance to LANL's Science and Technology Base and National R\&D Needs}

This work is related to technology development at Los Alamos and at other US facilities. Our study of $\mathrm{x}$-ray and gamma-ray emission from neutron stars and other compact objects will allow scientists to interpret data from a host of new $\mathrm{x}$-ray and gammaray instruments designed to observe neutron stars. The Los Alamos ALEXIS satellite is continuously monitoring neutron stars over the whole sky in the extreme ultraviolet (EUV) through soft $x$-ray range.

The NASA-supported ROSAT satellite is observing thermal $x$-ray emission from the surfaces of several neutron stars. NASA's X-ray Timing Explorer will be launched in about one year to detect irregularities in the spin rates of neutron stars due to their superfluid interiors. The large European $\mathrm{x}$-ray satellite XMM will carry a contingent of instruments, including one from Los Alamos, to study faint and rapidly varying neutron stars. Our measurements of cosmic gamma rays, which will establish the definitive level of 
emission from discrete sources, will be the standard for evaluating the detectors of other groups.

\section{Scientific Approach and Accomplishments}

We have investigated the structure and evolution of neutron stars and black holes and the radiation emitted from these objects. We explored how the differential rotation between a neutron star crust and the more rapidly rotating interior superfluid leads to frictional heating that increases the star's observable surface emission. The frictional heating may reconcile rapid neutrino cooling from a deconfined quark core or direct-Urca emission with the observed surface temperatures from objects such as the Vela pulsar. We found that rapid neutrino emission produces a sudden plunge of a neutron star's surface temperature after 10 to 100 years, depending on the star's mass and radius. An observation of this phenomenon, coupled with an estimate of the neutron star's mass, yields a tight estimate of the stellar radius and hence the equation of state at supranuclear densities. Using the CYGNUS air shower array at Los Alamos, one of the world's premier detectors of astrophysical gamma-rays above $50 \mathrm{TeV}$, we searched for ultra-highenergy (UHE) emission from a number of northern pulsars, and other astrophysical objects; no sources were unambiguously detected. We have continued monitoring the putative pulsar in the remnant of supernova 1987A. The low emissivity and rapid period change of this object suggest it is a low-magnetic-field neutron star with a large oblateness.

We have studied the variability and spectra of radiation from neutron stars with the goal of inferring the physics of dynamical processes in the interiors of these dense stars and on their surfaces. We have found a surprising universality in several neutron star phenomena including soft-repeating gamma-ray bursts and pulsar spin irregularities. These phenomena exhibit statistical properties reminiscent of earthquakes, suggesting a seismic origin. We refined our statistical analyses of these phenomena using waiting time distributions and time series correlations. We developed models in which energy is stored and released in the crusts of neutron stars. We have employed variations of the slip-stick models that have proven successful in interpreting terrestrial phenomena. In our models the ultimate source of energy for soft-repeating gamma-ray bursts is the stellar magnetosphere. The magnetic field drives large-scale crustal motions that increase the multipolar components of the magnetosphere, decreasing its overall energy. The gamma radiation could result from magnetic field annihilation and the acceleration of high energy electrons and other particles. For the pulsar timing noise, the seismic activity is driven by 
the decreasing rotation rate of the star and its changing equilibrium figure. Fluctuations in the stellar moment of inertia create the observed noise in the pulsar spin rates.

\section{Publications}

1. D. E. Alexandreas, D. Berley, S. D. Biller, G. M. Dion, J. A. Goodman, T. J. Haines, C. M. Hoffman, E. P. Horch, X-Q. Lu, C. Sinnis, G. B. Yodh, and W. Zhang, "Point Source Search Techniques in Ultra High Energy Gamma Ray Astronomy," Nucl. Instrum. Methods A 328, 570 (1993).

2. D. E. Alexandreas, G. Allen, D. Berley, S. Biller, R. L. Burman, D. R. Cady, M. Cavalli-Sforza, C. Y. Chang, D. Coyne, C. Dion, G. M. Dion, D. Dorfan, R. W. Ellsworth, S. J. Freedman, B. K. Fujikawa, J. A. Goodman, T. J. Haines, C. M. Hoffman, L. Kelley, S. Klein, D. A. Krakauer, P. W. Kwok, X- Q. Lu, D. E. Nagle, M. Potter, V. D. Sandberg, C. Sinnis, A. Shoup, M. J. Stark, P. R. Vishwanath, D. D. Weeks, D. A. Williams, J-P. Wu, G. B. Yodh, and W. Zhang, "Daily Search for Emission of Ultra High Energy Radiation from Point Sources," Astrophys. J. Lett. 405, 353 (1993).

3. D. E. Alexandreas, G. E. Allen, D. Berley, S. Biller, R. L. Burman, M. CavalliSforza, C. Y. Chang, M. L. Chen, P. Chumney, D. D. Coyne, C. Dion, G. M. Dion, D. Dorfan, R. W. Ellsworth, J. A. Goodman, T. J. Haines, C. M. Hoffman, L. Kelley, S. Klein, D. E. Nagle, S. C. Schaller, R. Schnee, C. Sinnis, A. Shoup, M. J. Stark, D. D. Weeks, D. A. Williams, J.-P. Wu, T. Yang, G. B. Yodh, and W. Zhang, "Search for Emission of Ultra-High-Energy Radiation from Active Galactic Nuclei," Astrophys. J. 418, 832 (1993).

4. D. E. Alexandreas, G. E. Allen, D. Berley, S. Biller, R. L. Burman, M. CavalliSforza, C. Y. Chang, M.-L. Chen, P. Chumney, D. Coyne, C. L. Dion, G. M. Dion, D. Dorfan, R. W. Ellsworth, J. A. Goodman, T. J. Haines, M. Harmon, C. M. Hoffman, L. Kelley, S. Klein, D. E. Nagle, D. M. Schmidt, R. Schnee, C. Sinnis, A. Shoup, M. J. Stark, D. D. Weeks, D. A. Williams, J.- P. Wu, T. Yang, G. B. Yodh, and W. P. Zhang, "A New Limit on the Rate- Density of Evaporating Black Holes," Phys. Rev. Lett. 71, 2524 (1993).

5. S. Biller, D. E. Alexandreas, G. E. Allen, D. Berley, R. L. Burman, M. CavalliSforza, C. Y. Chang, M. L. Chen, P. Chumney, D. D. Coyne, C. Dion, G. M. Dion, D. Dorfan, R. W. Ellsworth, J. A. Goodman, T. J. Haines, T. J. Harmon, C. M. Hoffman, L. Kelley, S. Klein, D. E. Nagle, S. C. Schaller, D. M. Schmidt, R. Schnee, A. Shoup, C. Sinnis, M. J. Stark, D. D. Weeks, D. A. Williams, J.-P. Wu, T. Yang, G. B. Yodh, and W. Zhang, "Search for Ultra-High-Energy Point-Source Emission over Various Timescales," Astrophys. J. 423, 714 (1994)

6. D. E. Alexandreas, G. E. Allen, D. Berley, S. Biller, R. L. Burman, M. CavalliSforza, C. Y. Chang, M.-L. Chen, P. Chumney, D. Coyne, C. L. Dion, G. M. Dion, D. Dorfan, R. W. Ellsworth, J. A. Goodman, T. J. Haines, M. Harmon, C. M. Hoffman, L. Kelley, S. Klein, D. E. Nagle, S. C. Schaller, D. M. Schmidt, R. Schnee, A. Shoup, C. Sinnis, M. J. Stark, D. D. Weeks, D. A. Williams, J.-P. Wu, T. Yang, G. B. Yodh, and 
W. P. Zhang, "Search for Ultra-High-Energy Radiation from Gamma-Ray Bursts," Astrophys. J. Lett. 426, L1 (1994).

7. V. Urpin and K. Van Riper, "Crustal Magnetic Field Decay in Neutron Stars and the Nature of Superdense Matter," Astrophys. J. (Letters) 411, 87, 1993.

8. J. M. Lattimer, K. A. Van Riper, M. Prakash, and M. Prakash, "Rapid Cooling and the Structure of Neutron Stars," Astrophys. J. 425, 802, 1994.

9. J. A. Miralles, K. A. Van Riper, and J. M. Lattimer, "The Boltzmann Equation in General Relativistic Rotating Systems: Cooling of Rotating Neutron Stars," Astrophys. J. 407, 687, 1993.

10. B. Link, R. I. Epstein, and G. Baym, "Superfluid Vortex Creep and Rotational Dynamics of Neutron Stars," Astrophys. J. (Letters) 403, 285, 1993.

11. K. Chen, J. Middleditch, and M. Ruderman, "Low-Mass X-Ray Binaries and Millisecond Pulsars in Globular Cluster," Astrophys. J. (Letters) 408, L17, 1993.

12. W. T. S. Deich, J. Middleditch, S. B. Anderson, P. Gorham, S. R. Kulkarni, and T. A. Prince, "The Binary Pulsar PSR 1908+00 in NGC 6760," Astrophys. J. (Letters) 410, 95, 1993.

13. D. E. Alexandreas et al., "Daily Search for Emission of Ultra High Energy Radiation from Point Sources," Astrophys. J. 405, 353, 1993.

14. D. E. Alexandreas et al., "Point-Source Search Techniques in Ultra-High-Energy Gamma Ray Astronomy," Nucl. Instrum. Methods A 328, 570 (1994).

15. D. E. Alexandreas et al., "Search for Ultra-High-Energy Point-Source Emission over Various Timescales," Astrophys. J. 423, 714 (1994).

16. D. E. Alexandreas et al., "Search for Ultra-High-Energy Emission from GammaRay Bursts," Astrophys. J. Lett. 426, 1 (1994).

17. J. M. Lattimer et al., "Rapid Cooling and the Structure of Neutron Stars," Astrophys. J. 425, 802 (1994).

18. K. A. Van Riper, B. Link, and R. I. Epstein, "Frictional Heating and Neutron Star Thermal Evolution," Astrophys. J 448, 294 (1995). 